\title{
BMJ Open MIS-TLIF versus O-TLIF for single-level degenerative stenosis: study protocol for randomised controlled trial
}

\author{
Olga N Leonova (D) , ${ }^{1}$ Evgeny A Cherepanov, ${ }^{2}$ Aleksandr V Krutko ${ }^{3}$
}

To cite: Leonova ON, Cherepanov EA, Krutko AV. MISTLIF versus 0 -TLIF for singlelevel degenerative stenosis: study protocol for randomised controlled trial. BMJ Open 2021;11:e041134. doi:10.1136/ bmjopen-2020-041134

- Prepublication history for this paper is available online. To view these files, please visit the journal online (http://dx.doi org/10.1136/bmjopen-2020041134).

Received 31 May 2020 Revised 17 February 2021 Accepted 17 February 2021

\section{Check for updates}

(c) Author(s) (or their employer(s)) 2021. Re-use permitted under CC BY-NC. No commercial re-use. See rights and permissions. Published by BMJ.

${ }^{1}$ Neurosurgery Department, Novosibirsk Research Institute of Traumatology and Orthopaedics, Novosibirsk, Russian Federation ${ }^{2}$ Project Management, Biorasi, Aventura, Florida, USA

${ }^{3}$ Neurosurgery Department,

Priorov National Medical

Research Center of

Traumatology and Orthopedics, Moscow, Russian Federation

Correspondence to

Dr Olga N Leonova;

onleonova@gmail.com

\section{ABSTRACT}

Introduction Patients with symptomatic single-level combination of degenerative stenosis and low-grade spondylolisthesis are often treated by nerve root decompression and spinal fusion. The gold standard is traditional open decompression and fusion, but minimally invasive method is more and more prevailing. However, there is lack of high-quality studies comparing these two techniques in order to obtain the advantages and certain indications to use one of these methods. The current study includes clinical, safety and radiological endpoints to determine the effectiveness of minimally invasive decompression and fusion (MIS-TLIF) over the traditional open one (0-TLIF).

Methods and analysis All patients aged 40-75 years with neurogenic claudication or bilateral radiculopathy caused by single-level combination of degenerative stenosis and low-grade spondylolisthesis, confirmed by MRI with these symptoms persisting for at least 3 months prior to surgery, are eligible. Patients will be randomised into MIS-TLIF or traditional 0-TLIF. The primary outcome measure is 0swestry Disability Index at 3-month followup term. The secondary outcomes are patient-reported outcome measures by the number of clinical scales, radiological parameters including sagittal balance parameters, safety endpoints and cost-effectiveness of each method. All patients will be analysed preoperatively, as well as on the 14th day of hospital stay (or on the day of hospital discharge), 3 months, 6 months, 12 months and 24 months postoperatively. The study has the design of a parallel group to demonstrate the non-inferior clinical results of MIS-TLIF compared with the traditional 0-TLIF. Ethics and dissemination The study will be performed according to Helsinki Declaration. The study protocol was approved by the Local Ethical Committee of Priorov National Medical Research Center of Traumatology and Orthopedics in August 2020. Preliminary and final results will be presented in peer-reviewed journals, especially orthopaedic and spine surgery journals, at national and international congresses.

Trial registration number NCT04594980.

\section{INTRODUCTION}

Lumbar spinal stenosis is a pathological process where bony, ligamentous and synovial elements of the lower axial spine degenerate and overgrow, progressively compressing the neural and vascular elements in the spinal

\section{Strengths and limitations of this study}

- Design of non-inferiority shows comparative clinical effectiveness of two methods for the primary endpoint and the superiority of one of the methods on secondary endpoints (functional outcomes, radiological and safety).

- The primary endpoint at 3-month term was chosen due to the greatest regression of Oswestry Disability Index that occurs at this time compared with the baseline.

- Indications for surgical treatment and outcome assessment will take into account sagittal balance parameters by Gille.

- The patient cohort is represented by homogeneous patients, suffering single-level combination of degenerative stenosis and low-grade spondylolisthesis.

- Cost-effectiveness estimation should be extrapolated with caution because there may be significant cost differences with other regions and countries.

canal. ${ }^{1}$ Degenerative spondylolisthesis is one of the common disorders in lumbar spine region, and often results in lumbar stenosis, with symptoms of lower back pain, leg pain, neurogenic claudication and decreased function. ${ }^{2}$

According to the meta-analysis, the prevalence of clinical symptoms of lumbar stenosis is found in $11 \%$ in the overall population and in $39 \%$ in patients with back pain. ${ }^{3}$ Population radiographic examination of adults older than 40 years old showed that the prevalence of moderate stenosis is from $23.6 \%$ to $77.9 \%$; severe stenosis is to be found in $8.4 \%-30.4 \%$ of patients. ${ }^{1}$

A total of $88.9 \%$ patients with the primary diagnosis of lumbar spinal stenosis undergo surgical treatment. ${ }^{4}$ The performance of decompression is necessary to release compressed neural structures; specifically, decompression is performed from targeted resection of compressing structure to wide laminectomy. To a considerable degree, the volume of decompression is based on clinical assessment of each concrete case. 
The segment instability after decompression remains one of the main causes of performing fusion, all the more so that the presence of preceding low-grade spondylolisthesis is associated with increased risk of instability and performing the following segment fusion. ${ }^{5}$ The surgical treatment of degenerative lumbar stenosis and degenerative spondylolisthesis demonstrates clinically important success 2, 4 and 8 years postoperatively. ${ }^{6}$ Thus, decompression in case of spondylolisthesis requires fusion performing.

Open surgery provides a larger volume of decompression according to MRI scans, than minimally invasive procedure, though without statistical significance from the clinical point of view. ${ }^{7}$ Minimally invasive decompression is still questioned due to a limited surgical view and space, long learning curve of surgeons, increased X-ray radiation for both patients and surgeons, ${ }^{2}$ and often due to longer duration of a surgical session. ${ }^{8}$

According to the systematic review, the patients report about equivalent clinical results of open (O-TLIF) and minimally invasive decompression and fusion (MISTLIF), ${ }^{9}{ }^{10}$ with similar ${ }^{11}$ or a fewer number of postoperative complications after minimally invasive fusion. ${ }^{12}$ However, all the authors claim, that most part of the articles, forming the foundations of their reviews, have the third and the fourth levels of evidence, basing on heterogeneous patient cohorts, invalid endpoints and the following low-quality analysis of data.

According to the other systematic reviews, including only randomised clinical trials (RCTs) $(n=7)$, MIS-TLIF was associated with lower blood loss, a shorter stay in hospital and somewhat lower disability in the interim period of monitoring, longer time of X-ray. ${ }^{13}$ There were no observations of statistically significant group differences in the time of the procedure, perioperative complications, pseudarthrosis or pain severity in the back/leg. ${ }^{13}$ However, only two of seven RCTs, included into the analysis, analyse spondylolisthesis exclusively. Furthermore, in spite of the urgency of the issue, both RCTs have high bias risk (unreliable randomisation, the study design is not indicated, there are no calculations of the sample size). That is why, there is no basis to claim that the effectiveness of minimally invasive or open technology in the treatment of degenerative lumbar stenosis is a settled question.

Despite potential advantages, it remains unclear, if MISTLIF is consistent with traditional O-TLIF in the treatment of combination of degenerative stenosis and low-grade spondylolisthesis. To solve this issue, it is necessary to carry out high-quality randomised research, reporting comparative effectiveness of these two methods.

\section{Research aim and objectives}

The aim of the current study is to determine the effectiveness of the MIS-TLIF over the O-TLIF in patients with single-level combination of degenerative lumbar stenosis and low-grade spondylolisthesis by comparing the clinical efficacy and safety.

\section{METHODS AND ANALYSIS}

\section{Patient and public involvement}

Patients and/or the public were not involved in the design, or conduct, or reporting, or dissemination plans of this research.

\section{Study design and settings}

The current study is an open-label single-centre randomised controlled trial in which 96 patients with symptomatic single-level combination of degenerative stenosis and low-grade spondylolisthesis will be allocated into MIS-TLIF versus traditional O-TLIF. All patients included in the current study will be treated at the Priorov National Medical Research Center of Traumatology and Orthopedics, Russia. The study has the design of a parallel group to demonstrate the non-inferior clinical results of MIS-TLIF compared with the traditional O-TLIF. The study is registered at http://www.clinicaltrials.gov, which can be accessed online.

The presented protocol follows the recommendations outlined in the Standard Protocol Items: Recommendations for Interventional Trials guidelines for randomised controlled trials.

\section{Patient selection}

All patients aged 40-75 years with neurogenic claudication or bilateral radiculopathy caused by single-level combination of degenerative stenosis and low-grade spondylolisthesis confirmed on MRI with these symptoms persisting for at least 3 months prior to surgery will be enrolled in the current study. A full list of inclusion and exclusion criteria is given in table 1 .

\section{Randomisation}

Subjects who meet the inclusion criteria after oral and written consent (see online supplemental file 1 'Informed Consent Form') will be randomised into one of two arms, minimally invasive surgery procedure and traditional open surgery, with 1:1 ratio. The allocation of patients into groups will be conducted by stratified block randomisation to guarantee balance of patients with stenosis grade ( $\mathrm{B}$ or $\mathrm{C}$ by Shizas $e t a l^{14}$ ) and grading of nerve root compression (by Pfirrmann et als sRI-based system ${ }^{15}$ ). A randomised block design will be stratified using a computer-generated randomisation scheme maintained by a centralised randomisation centre. The list of randomisation is available only to those independent remote study team members, who will not participate in other activities involving study patients. A predefined block size will be used to ensure balanced group sizes at the end of the inclusion period. Randomisation will be conducted on visit 2 (baseline) 1-3 days before surgery by one designated team member.

\section{Baseline data}

The baseline data will include data from demographic and comorbidity variables, clinical scores of Oswestry Disability Index (ODI), the Numeric Pain Rating Scale for low back and leg pain (NPRS), the EuroQol 
Table 1 Inclusion and exclusion criteria

\begin{tabular}{ll} 
Inclusion criteria & Exclusion criteria \\
\hline Age from 40 to 75 years & Bilateral foraminal stenosis requiring surgical decompression on \\
Neurogenic claudication or bilateral radiculopathy & both sides \\
caused by single-level combination of degenerative & Degenerative spondylolisthesis $2 \mathrm{~B}, 3$ subtypes by Gille \\
stenosis and spondylolisthesis confirmed by MRI at & Spondylolisthesis grade II or higher by Meyerding of any aetiology \\
one level L3-L4 or L4-L5 or L5-S1 & More than one symptomatic level requiring multilevel surgical \\
Symptoms persisting for at least 3 months prior to & decompression and/or fusion \\
surgery & Any condition that cannot be treated with mini-invasive unilateral \\
Given written informed consent form & decompression and fusion \\
Be able and agree to fully comply with the clinical & Any contraindication or inability to undergo baseline and/or follow- \\
protocol and willing to adhere to follow-up schedule & up MRI or X-ray as required per protocol \\
and requirements & Prior lumbar spinal fusion at any level \\
Oswestry Disability Index score of at least 40/100 at & Other non-degenerative spinal conditions that may have an impact \\
baseline & on subject safety, well-being or the intent and conduct of the study \\
& History or presence of any other major neurological disease or \\
& condition that may interfere with the study assessments
\end{tabular}

Five-Dimensional descriptive system questionnaire (EQ5D), Douleur Neuropathique 4 (DN4), the Health Transition Item from SF-36 (HTI Item) and Clinical Global Impression of Change (CGIC).

Radiological assessment will include X-ray at the step mode, MRI and CT of the lumbar spine. X-rays will be performed at step mode (from C1 vertebra to the femoral heads standing) in neutral anterior-posterior and lateral positions. The MRI scan will present T1-weighted and T2-weighted axial and sagittal images of the lumbar spine. A CT scan will be performed with a low-dose multiplane 64-slice tomography of the lumbar spine.

\section{Surgical procedure}

All patients in both groups will be operated with the aid of spinal navigation C-arm. The surgical procedure will be performed after the patient is put under general anaesthesia. All participating surgeons have performed at least 30 procedures of each-MIS-TLIF and O-TLIF-prior to the start of the trial. The use of a posterior screw fixation system is mandatory in both groups. The interbody graft represents polyetheretherketone cages filled by autologous bone chips.

In case of MIS-TLIF, unilateral paramedian skin incision is used $(3-5 \mathrm{~cm})$ for exposing facet joints and the implantation of pedicle screws from the side of approach. In case of O-TLIF, a long incision will be made in midline $(10-15 \mathrm{~cm})$ with the following separation of paraspinal muscles from the midline and the implantation of pedicle screws from both sides. Then decompression will be performed. In case of MIS-TLIF, unilateral total facetectomy will be performed, which is necessary for performing TLIF too. Then using the same side approach, the recalibration of the spine canal over the top to the opposite side will be provided, that is, partial medial facetectomy at the opposite side will be performed. In case of O-TLIF, laminectomy with bilateral facetectomy will be performed for decompression. After performing decompression and removing the causes of stenosis, the following implantation of interbody cage with feasible restoration of a segmental angle will be carried out. In case of MIS-TLIF, the implantation of screws percutaneously will be the final stage on the opposite side.

\section{Outcome measurements \\ Primary outcome}

The primary outcome will be the ODI change at the 3-month follow-up comparing with baseline. The Oswestry scale is widely used for patients with spine disease and is a reliable and proven method for assessing the functional capacity of patients. ${ }^{1617}$ In this study, we will use the current V.2.1a translated and validated in Russian. The Oswestry scale consists of 10 questions, each is offered six answers, the answer option is rated from 0 to 5 points. Then the resulting points are converted to percentages, which allow estimating how pain in the back and/or leg affects the patient's daily life.

\section{Secondary outcome}

One of the secondary outcomes is NPRS, assessing the back and leg pain in rest without taking any tests (ranging from 0 to $100 \mathrm{~mm}$ ) in follow-up terms. NPRS is represented as a reliable and validity scale. Pain will be rated on a horizontal scale of $100 \mathrm{~mm}$, ranging from $0 \mathrm{~mm}$, 'no pain', to $100 \mathrm{~mm}$, 'the worst pain you can imagine'. Patients do not see the results of previous assessments and assess the pain that occurred during the visit.

EQ-5D is a standardised instrument for measuring generic health status. It complements the standard set of the International Consortium for Health Outcomes Measurement for low back pain. EQ-5D is currently used in spine surgery practice due to its commitment, easy 
usage in practice. The questionnaire has shown its reliability, validity and responsiveness. ${ }^{18} 19$

DN4 allows to identify the neuropathic components of a chronic pain syndrome. The questionnaire is easy to use for patients and for the investigator too. With clarifying the pain traits, the investigator can suspect and identify the neuropathic origin of the pain syndrome. The questionnaire can indicate neuropathic pain with sufficient sensitivity and specificity. ${ }^{20} 21$

The HTI Item is one question from the SF-36 survey. ${ }^{22} 23$ Moreover, assuming the correlation of HTI Item of the patient and the clinician, we want to get an objective assessment of the patient's condition and satisfaction. The answers range from 'Much Better', 'Somewhat Better', 'About the Same', 'Somewhat Worse', to 'Much Worse'.

The CGIC scale will be used to measure changes in the patient's condition after the surgical treatment according to the investigator's opinion. ${ }^{24}$ The patient's condition will be assessed on a CGIC scale by an experienced clinician who is familiar with the disease and the likely progress of treatment. The clinician makes a judgement about the overall picture of the patient at each visit, comparing with the baseline. We will use a modified 5-point scale assessing the present patient's condition: 1 -very much improved, 2-somewhat improved, 3-no changes, 4middle worse, 5 -very much worse.

Cost-effectivenessof the hospital stay will be evaluated by summarising cost of surgical procedure (implanted system, the salary for surgery team) and the cost of the number of days before discharge. The cost of the additional procedures in case of complications will be added to the total cost of the patient's hospital stay.

The assessment of interbody fusion rate success will be evaluated on CT scans at 12-month and 24-month follow-up. Using the method described by Tan et al, ${ }^{25}$ the patients will be graded into one of four grades of fusion-grade I (complete fusion) to grade IV (bipolar pseudarthrosis).

Sagittal balance parameters will be evaluated on X-ray; including pelvic incidence, pelvic tilt, global lordosis angle, segmental lordosis and sagittal vertical axis. The sagittal balance is a physiological alignment of the spine in the most efficient manner by the muscular forces; parameters help to guide the surgical strategy in spinal surgery. It is very useful in preoperative planning, but allows also to understand what went biomechanically wrong after a surgery.

Safety endpoints such as blood loss, surgery's duration, duration of hospital stay, surgical complications and the incidence of reoperations will be documented in all patients.

\section{Data collection, management and analysis}

All patients will be analysed preoperatively, as well as on the 14th day of hospital stay or on the day of hospital discharge (depends on what event comes first), 3 months, 6 months, 12 months and 24 months postoperatively, according to the assessment schedule (table 2).
The data from initial visits, hospital staying and follow-up visits will be fixed into a database via an electronic data capture system. The data will be recorded and analysed without any personal identifiers by using coded information. The source documents and identifiers will be archived in a security facility and permission for accessing data will be documented by the investigator. The study will be monitored by the internal monitor to ensure quality of the data in accordance with established principles of Good Clinical Practice.

The intention-to-treat (ITT) population analysis, which consists of patients' violated protocol, due to its poor conduct, helps to prove non-inferior. The per-protocol population analysis, which population includes ideal patients, is not representative for the whole study population. ${ }^{26}$ So, all efficacy analyses will be conducted on modified ITT (mITT) population, which is defined as all randomised patients who complete the surgical procedure, and completed at least with one post-discharge evaluation visit. In addition, it will be the term for the primary endpoint. In analyses based on the mITT population, subjects will be analysed according to their randomised treatment assignment.

\section{Statistical analysis}

An exploratory data analysis will be conducted: the identification of misprints, the investigation of normality by the Shapiro-Wilk test, the detection of outliers, the construction of histograms and distribution plots.

The comparison of continuous data between the groups will be conducted using the unpaired Mann-Whitney $\mathrm{U}$ test with the calculation of the distribution bias. The comparison of continuous data within the group will be conducted by the paired Mann-Whitney $\mathrm{U}$ test with the calculation of the distribution bias.

The comparison of categorical and binary data between groups will be conducted by Fisher's exact two-sided test. The comparison of categorical and binary data within group will be conducted by the McNemar's test. For complications, the Kaplan-Meier risk curves will be plotted. The comparison of risks of complications between groups will be conducted by the log-rank test.

The investigation of pairwise relationships between continuous data and ODI will be conducted by calculating the Spearman correlation coefficients. The investigation of multidimensional relations between continuous data and ODI will be conducted by constructing a generalised multifactorial linear regression.

Finding individual and multiplicative predictors of complications will be conducted by constructing singlefactor and multivariate logistic regressions, respectively. The predictive quality of the resulting logistic regression models will be evaluated using receiver operating characteristic analysis methods.

Testing statistical hypotheses will be conducted at a critical level of significance $\mathrm{p}=0.05$. The lower limit of the proof power is fixed at $80 \%$. 
Table 2 Summary of scheduled procedures and assessments

\begin{tabular}{|c|c|c|c|c|c|c|c|c|}
\hline & $\begin{array}{l}\text { Visit } 1 \\
\text { screening }\end{array}$ & $\begin{array}{l}\text { Visit } 2 \\
\text { baseline }\end{array}$ & $\begin{array}{l}\text { Visit } 3 \\
\text { day of } \\
\text { surgery }\end{array}$ & $\begin{array}{l}\text { Visit } 4 \\
\text { hospital } \\
\text { discharge } \\
\text { or 14th day } \\
\text { of hospital } \\
\text { stay }\end{array}$ & $\begin{array}{l}\text { Visit } 5 \\
\text { 3-month } \\
\text { follow-up }\end{array}$ & $\begin{array}{l}\text { Visit } 6 \\
\text { 6-month } \\
\text { follow-up }\end{array}$ & $\begin{array}{l}\text { Visit } 7 \\
\text { 12- month } \\
\text { follow-up }\end{array}$ & $\begin{array}{l}\text { Visit } 8 \\
24 \\
\text { months } \\
\text { end of } \\
\text { study }\end{array}$ \\
\hline $\begin{array}{l}\text { Visit window } \\
\text { ( } \pm \text { days })\end{array}$ & & & & & $90 \pm 14$ & $183 \pm 14$ & $365 \pm 28$ & $730 \pm 56$ \\
\hline Informed consent & $x$ & & & & & & & \\
\hline Demographics & $x$ & & & & & & & \\
\hline Medical history & $x$ & & & & & & & \\
\hline $\begin{array}{l}\text { Indications for } \\
\text { surgery }\end{array}$ & $x$ & & & & & & & \\
\hline Eligibility criteria & $x$ & & & & & & & \\
\hline ODI & $x$ & & & $x$ & $x$ & $x$ & $x$ & $x$ \\
\hline NPRS & $\mathrm{x}$ & & & $x$ & $\mathrm{x}$ & $\mathrm{x}$ & $x$ & $x$ \\
\hline EQ-5D & $x$ & & & $x$ & $x$ & $x$ & $x$ & $x$ \\
\hline DN4 & $x$ & & & $x$ & $x$ & $x$ & $x$ & $x$ \\
\hline $\begin{array}{l}\text { HTI Item (from } \\
\text { SF-36) }\end{array}$ & & & & $x$ & $x$ & $\mathrm{x}$ & $\mathrm{x}$ & $x$ \\
\hline CGIC & & & & $x$ & $x$ & $x$ & $x$ & $x$ \\
\hline Randomisation & & $x$ & & & & & & \\
\hline $\begin{array}{l}\text { Surgical } \\
\text { procedure }\end{array}$ & & & $x$ & & & & & \\
\hline X-ray & $x$ & & & $x$ & & & $x$ & $x$ \\
\hline MRI & $x$ & & & & & $x$ & $x$ & $x$ \\
\hline CT & $x$ & & & & & & $x$ & $x$ \\
\hline $\begin{array}{l}\text { Adverse events } \\
\text { (incl. device } \\
\text { related)/serious } \\
\text { adverse events }\end{array}$ & $\mathrm{X}$ & $x$ & $x$ & $\mathrm{x}$ & $x$ & $\mathrm{X}$ & $x$ & $x$ \\
\hline
\end{tabular}

CGIC, Clinical Global Impression of Change; DN4, Douleur Neuropathique 4; EQ-5D, EuroQol Five-Dimensional descriptive system questionnaire; HTI Item, Health Transition Item from SF-36; NPRS, Numeric Pain Rating Scale; ODI, Oswestry Disability Index.

\section{Sample size}

Under non-inferiority design, the sample size was calculated for ODI difference between the values before intervention and 3 months after. Assuming mean of ODI difference is 39 and SD of ODI difference is 19.3 in both groups (based on our own published data (18.8 at 1-year follow-up, ${ }^{27} 19.3$ at 3-month follow-up ${ }^{28}$ ), the noninferiority margin $\delta=12$, for a one-sided Mann-Whitney $\mathrm{U}$ test with a critical significance $\mathrm{p}=0.05$ and test power is $80 \%$, it is enough to allocate 38 patients. Considering the $20 \%$ loss, the total sample size will be 96 patients, 48 in each group.

The exact number of patients might be adjusted based on actual dropout rate achieved by the time of interim analysis.

\section{Adverse and serious adverse events}

During the complete study period, all adverse events will be reported. Adverse events are defined as any undesirable experience occurring to a participant, whether or not related to the intervention. The list of adverse effects is: adjacent segment disease, adjacent segment instability, screw loosening, implant migration, cage subsidence, pseudarthrosis, surgical site infection, worsening neurological symptoms, pain recurrence, dural tears and durotomy.

\section{DISCUSSION}

The current study is designed to have high evidence level, comparing the clinical effectiveness of the two most popular techniques of lumbar spine surgery: MIS-TLIF and traditional O-TLIF.

The non-inferiority design was chosen because we assume non-inferior (comparable) clinical efficacy on the primary endpoint and superiority on some secondary endpoints such as functional outcomes, safety or costeffectiveness, ${ }^{19}$ and fewer adverse events to patients. ${ }^{11} 29$ 
This formulation is fully described by non-inferiority trial design. ${ }^{30} 31$

There is no commonly accepted non-inferiority margin size available in the literature for clinical trials with the same population, study treatment and primary endpoint. We used minimally clinical important difference (MCID) published for ODI to determine the size of non-inferiority margin. According to various sources, the MCID range for ODI varies between 6.8 and 15 points. The reasons for such variability are the lack of placebo-controlled RCTs or trials with sham surgery as a comparator, high heterogeneity of study populations and follow-up periods. The difference between the values of MCID may be due to different methods of calculation. ${ }^{32}$ The highest value is a US Food and Drug Administration recommendation (minimum 15-point change in spinal fusion patients before surgery and at follow-up) which is referred to as a personal communication. ${ }^{33}$ The minimal MCID equal to 6.8 at 2-year follow-up is determined on 50 patients who underwent extension of fusion for adjacent-segment disease. Other estimations are 12 points (lumbar stenosis, 2-year follow-up), ${ }^{34} 14.19$ points (low-grade degenerative lumbar spondylolisthesis-associated back and leg pain, 2-year follow-up ${ }^{35}$ ), 12.54 points (lumbar stenosis, 1-year follow-up ${ }^{36}$ ) and 12.8 points (a general lumbar spine surgery population, the largest available study with 454 patients, however the exact indication for surgery is not specified, 1-year follow-up ${ }^{22}$ ). As far as there is no consensus in the literature of a certain value comparing mean ODI in groups, we determined the MCID for ODI as 12 points as more conservative value and as a mean value for relevant range.

The term of primary outcome analysis as 3 months was chosen due to the fact that the greatest regression of ODI compared with the baseline occurs at this stage. When analysing at a later term of follow-up, the regression is no longer so pronounced and less statistically significant. ${ }^{37} 38$ Comparing MIS-TLIF and O-TLIF, the ODI was significantly better in the minimally invasive group at 3 and 6 months after surgery, and the difference gradually diminished over time; so, there was no difference between the two groups at 12 and 24 months after surgery. ${ }^{39}$

This study is the one of the very few with level 1 of evidence about comparing the two most popular surgical methods in the treatment of single-level combination of degenerative lumbar stenosis and low-grade spondylolisthesis. We are planning to get a homogeneous patient cohort to compare two methods of surgical treatment. That is why the results of the current study can be the basis for spinal surgery guidelines: the definition of clear indications for the application of minimally invasive and traditional open procedure in lumbar stenosis will help to create an algorithm for surgical treatment and patient selection, thus adding to the existent body of evidence.

\section{ETHICS AND DISSEMINATION}

The study will be performed according to Helsinki Declaration. The study protocol was approved by the Local Ethical Committee of Priorov National Medical Research Center of Traumatology and Orthopedics in August 2020. Preliminary and final results will be presented in peer-reviewed journals, especially orthopaedic and spine surgery journals, at national and international congresses.

Contributors ONL and EAC contributed to the study concept and wrote the study protocol. AVK designed the study protocol, is the primary investigator and coordinator of the study. ONL, EAC and AVK drafted the manuscript, critically reviewed and approved the final manuscript.

Funding The authors have not declared a specific grant for this research from any funding agency in the public, commercial or not-for-profit sectors.

Competing interests None declared.

Patient consent for publication Not required.

Provenance and peer review Not commissioned; externally peer reviewed.

Supplemental material This content has been supplied by the author(s). It has not been vetted by BMJ Publishing Group Limited (BMJ) and may not have been peer-reviewed. Any opinions or recommendations discussed are solely those of the author(s) and are not endorsed by BMJ. BMJ disclaims all liability and responsibility arising from any reliance placed on the content. Where the content includes any translated material, BMJ does not warrant the accuracy and reliability of the translations (including but not limited to local regulations, clinical guidelines, terminology, drug names and drug dosages), and is not responsible for any error and/or omissions arising from translation and adaptation or otherwise.

Open access This is an open access article distributed in accordance with the Creative Commons Attribution Non Commercial (CC BY-NC 4.0) license, which permits others to distribute, remix, adapt, build upon this work non-commercially, and license their derivative works on different terms, provided the original work is properly cited, appropriate credit is given, any changes made indicated, and the use is non-commercial. See: http://creativecommons.org/licenses/by-nc/4.0/.

ORCID iD

Olga N Leonova http://orcid.org/0000-0002-9916-3947

\section{REFERENCES}

1 Bagley C, MacAllister M, Dosselman L, et al. Current concepts and recent advances in understanding and managing lumbar spine stenosis. F1000Res 2019;8:137-9.

2 Wu A-M, Hu Z-C, Li X-B, et al. Comparison of minimally invasive and open transforaminal lumbar interbody fusion in the treatment of single segmental lumbar spondylolisthesis: minimum two-year follow up. Ann Transl Med 2018;6:105.

3 Jensen RK, Jensen TS, Koes B, et al. Prevalence of lumbar spinal stenosis in general and clinical populations: a systematic review and meta-analysis. Eur Spine J 2020;29:2143-63.

4 Al Jammal OM, Delavar A, Maguire KR, et al. National trends in the surgical management of lumbar spinal stenosis in adult spinal deformity patients. Spine 2019;44:E1369-78.

5 Guha D, Heary RF, Shamji MF. latrogenic spondylolisthesis following laminectomy for degenerative lumbar stenosis: systematic review and current concepts. Neurosurg Focus 2015;39:E9.

6 Ilyas H, Udo-Inyang I, Savage J. Lumbar spinal stenosis and degenerative spondylolisthesis: a review of the sport literature. Clin Spine Surg 2019;32:272-8.

7 Fu C-J, Chen W-C, Lu M-L, et al. Comparison of paraspinal muscle degeneration and decompression effect between conventional open and minimal invasive approaches for posterior lumbar spine surgery. Sci Rep 2020;10:1-8.

8 Chan AK, Bisson EF, Bydon M, et al. A comparison of minimally invasive and open Transforaminal lumbar interbody fusion for grade 1 degenerative lumbar spondylolisthesis: an analysis of the prospective quality outcomes database. Neurosurgery 2020;87:555-62.

9 Heary RF, Kaiser MG, Goldstein CL. Editorial: perioperative outcomes and adverse events of minimally invasive surgery during transforaminal lumbar interbody fusion/posterior lumbar interbody fusion. J Neurosurg Spine 2016;24:413-5. 
$10 \mathrm{Ka} \mathrm{K}, \mathrm{Ng} \mathrm{M}$, Pui J, et al. Is minimally invasive surgery superior to open surgery for treatment of lumbar spinal stenosis? A systematic review. J Orthop Surg 2017;25:1-11.

11 Phan K, Mobbs RJ. Minimally invasive versus open laminectomy for lumbar stenosis: a systematic review and meta-analysis. Spine 2016;41:E91-100.

12 Weiss H, Garcia RM, Hopkins B, et al. A systematic review of complications following minimally invasive spine surgery including Transforaminal lumbar interbody fusion. Curr Rev Musculoskelet Med 2019;12:328-39.

13 Miller LE, Bhattacharyya S, Pracyk J. Minimally invasive versus open Transforaminal lumbar interbody fusion for Single-Level degenerative disease: a systematic review and meta-analysis of randomized controlled trials. World Neurosurg 2020;133:358-65.

14 Schizas C, Theumann N, Burn A, et al. Qualitative grading of severity of lumbar spinal stenosis based on the morphology of the dural sac on magnetic resonance images. Spine 2010;35:1919-24.

15 Pfirmann CWA, Dora C, Schmid MR, et al. Mr image-based grading of lumbar nerve root compromise due to disk herniation: reliability study with surgical correlation. Radiology 2004;230:583-8.

16 Mannion AF, Junge A, Grob D, et al. Development of a German version of the Oswestry disability index. Part 2: sensitivity to change after spinal surgery. Eur Spine J 2006;15:66-73.

17 Gum JL, Carreon LY, Stimac JD, et al. Predictors of Oswestry disability index worsening after lumbar fusion. Orthopedics 2013;36:e478-83.

18 Pennington Z, Alentado VJ, Lubelski D, et al. Quality of life changes after lumbar decompression in patients with tandem spinal stenosis. Clin Neurol Neurosurg 2019;184:105455.

19 Austevoll IM, Gjestad R, Grotle M, et al. Follow-Up score, change score or percentage change score for determining clinical important outcome following surgery? an observational study from the Norwegian Registry for spine surgery evaluating patient reported outcome measures in lumbar spinal stenosis and lumbar degenerative spondylolisthesis. BMC Musculoskelet Disord 2019;20:1-15.

20 Gudala K, Ghai B, Bansal D. Usefulness of four commonly used neuropathic pain screening questionnaires in patients with chronic low back pain: a cross-sectional study. Korean J Pain 2017;30:51-8.

21 Attal N, Perrot S, Fermanian J, et al. The neuropathic components of chronic low back pain: a prospective multicenter study using the DN4 questionnaire. J Pain 2011;12:1080-7.

22 Copay AG, Glassman SD, Subach BR, et al. Minimum clinically important difference in lumbar spine surgery patients: a choice of methods using the Oswestry disability index, medical outcomes study questionnaire short form 36 , and pain scales. Spine $J$ 2008;8:968-74.

23 Parker SL, Mendenhall SK, Shau D, et al. Determination of minimum clinically important difference in pain, disability, and quality of life after extension of fusion for adjacent-segment disease. J Neurosurg Spine 2012;16:61-7.
24 Scott W, McCracken LM. Patients' Impression of Change Following Treatment for Chronic Pain: Global, Specific, a Single Dimension, or Many? The Journal of Pain 2015;16:518-26.

25 Tan GH, Goss BG, Thorpe PJ, et al. Ct-Based classification of long spinal allograft fusion. Eur Spine J 2007;16:1875-81.

26 Lesaffre E. Noninferiority clinical trials: the good, the bad, and the ugly. Semin Liver Dis 2018;38:097-102.

27 Krutko AV, Leonova ON, Cherepanov EA. The importance of psychological testing in predicting the effectiveness of lumbar spine surgery. Coluna/Columna 2018;17:227-32.

28 Krutko AV, Akhmetyanov SA, Kozlov DM, et al. Minimum invasive stabilizing and Decompressive-Stabilizing methods of surgical treatment for Degenerative-Dystrophic lesions of spine. Vestn Travmatol i Ortop Im NN Priorova 2011:№ 4. Pp. 3.

29 Nerland US, Jakola AS, Solheim O, et al. Minimally invasive decompression versus open laminectomy for central stenosis of the lumbar spine: pragmatic comparative effectiveness study. BMJ 2015;350:h1603.

30 Lestini WF, Fulghum JS, Whitehurst LA. Lumbar spinal fusion: advantages of posterior lumbar interbody fusion. Surg Technol Int 1994:3:577-90.

31 Vavken P. Rationale for and methods of superiority, noninferiority, or equivalence designs in orthopaedic, controlled trials. Clin Orthop Relat Res 2011.

32 Chung AS, Copay AG, Olmscheid N, et al. Minimum clinically important difference. Spine 2017;42:1096-105

33 Roland M, Fairbank J. The Roland-Morris disability questionnaire and the Oswestry disability questionnaire. Spine 2000;25:3115-24.

34 Försth P, Ólafsson G, Carlsson T, et al. A randomized, controlled trial of fusion surgery for lumbar spinal stenosis. N Engl J Med 2016;374:1413-23.

35 Parker SL, Adogwa O, Paul AR, et al. Utility of minimum clinically important difference in assessing pain, disability, and health state after transforaminal lumbar interbody fusion for degenerative lumbar spondylolisthesis. J Neurosurg Spine 2011;14:598-604.

36 Carreon LY, Bratcher KR, Canan CE, et al. Differentiating minimum clinically important difference for primary and revision lumbar fusion surgeries. J Neurosurg Spine 2013;18:102-6.

37 Lurie JD, Tosteson TD, Tosteson A, et al. Long-Term outcomes of lumbar spinal stenosis: eight-year results of the spine patient outcomes research trial (sport). Spine 2015;40:63-76.

38 Adogwa O, Elsamadicy AA, Han JL, et al. Do measures of surgical effectiveness at 1 year after lumbar spine surgery accurately predict 2-year outcomes? J Neurosurg Spine 2016;25:689-96.

39 Wang H-L, Lü FZ, Jiang J-Y, et al. Minimally invasive lumbar interbody fusion via mast quadrant retractor versus open surgery: a prospective randomized clinical trial. Chin Med J 2011;124:3868-74.

40 Gille O, Bouloussa H, Mazas S, et al. A new classification system for degenerative spondylolisthesis of the lumbar spine. Eur Spine $J$ 2017;26:3096-105. 